\title{
OPEN A novel approach of fabricating monodispersed spherical MoSiBTiC particles for additive manufacturing
}

\begin{abstract}
Zhenxing Zhou, Suxia Guo, Weiwei Zhou ${ }^{\bowtie}$ \& Naoyuki Nomura ${ }^{\bowtie}$
It is very challenging to fabricate spherical refractory material powders for additive manufacturing (AM) because of their high melting points and complex compositions. In this study, a novel technique, freeze-dry pulsated orifice ejection method (FD-POEM), was developed to fabricate spherical MoSiBTiC particles without a melting process. Elemental nanopowders were dispersed in water to prepare a high-concentration slurry, which was subsequently extruded from an orifice by diaphragm vibration and frozen instantly in liquid nitrogen. After a freeze-drying process, spherical composite particles with arbitrary composition ratios were obtained. The FD-POEM particles had a narrow size range and uniform elemental distribution. Mesh structures were formed within the FD-POEM particles, which was attributed to the sublimation of ice crystals. Furthermore, owing to their spherical morphology, the FD-POEM particles had a low avalanche angle of $42.6^{\circ}$, exhibiting good flowability. Consequently, the combination of FD-POEM and additive manufacturing has great potential for developing complex refractory components used in industrial applications.
\end{abstract}

Metallic alloys with superior mechanical and functional properties at elevated temperatures are in high demand in the aerospace industry ${ }^{1,2}$. Much attention has been paid to refractory intermetallic compounds in academic and industrial fields ${ }^{2,3}$. In the past decade, a particle-strengthened system of the MoSiBTiC type has been developed with a composition $65 \mathrm{Mo}-5 \mathrm{Si}-10 \mathrm{~B}-10 \mathrm{Ti}-10 \mathrm{C}$ (at\%) via arc-melting technique $\mathrm{e}^{4,5}$. This alloy had high creep strength at high temperatures ${ }^{6}$, good room-temperature fracture toughness $\left[\geq 15 \mathrm{MPa}(\mathrm{m})^{1 / 2}\right]^{7}$, and similar or even lower density compared to that of Ni-based superalloys $\left(\sim 8.8 \mathrm{~g} \mathrm{~cm}^{-3}\right)^{8,9}$. The MoSiBTiC alloys are considered as attractive structural materials in aerospace applications, owing to their high melting points and superior high-temperature mechanical properties ${ }^{10,11}$. However, it is difficult to manufacture refractory components with complex internal or external structures using traditional methods. Therefore, additive manufacturing (AM) is deemed as a possible alternative ${ }^{12}$.

Laser powder bed fusion (L-PBF) is one of newly developed AM techniques ${ }^{13,14}$. It utilizes a high-energy laser beam to selectively melt metallic powders to build three-dimensional objects in a layer-by-layer model ${ }^{15,16}$. Many factors influence the quality of the L-PBF fabricated parts. Among them, the properties of powders, such as their shape, size distribution, surface morphology, flowability, composition, and laser absorptivity, play a significant role. They directly influence the state of the melting pools, thereby affecting the quality of the final parts $^{17,18}$. Presently, the powders, including $\mathrm{Ti}^{-16,19}, \mathrm{Fe}_{-}^{-20}, \mathrm{Al}^{21,22}$ and $\mathrm{Co}$-alloys ${ }^{23}$, used for L-PBF, are mostly fabricated by mechanical milling, gas atomization (GA), plasma atomization (PA), plasma rotating electrode process (PREP), and plasma spheroidization (PS $)^{24,25}$. However, each of these methods has significant limitations in the preparation of refractory alloy powders. For instance, powders produced by mechanical milling always have some shortcomings, such as irregular shape, poor flowability, uncontrolled particle size, or contamination ${ }^{26}$. Zhou et al. fabricated MoSiBTiC powders for L-PBF via high-energy ball milling and sieving ${ }^{12,27}$. Although the powder size of $\sim 10-45 \mu \mathrm{m}$ was controlled, abundant internal defects such as cracks were formed in the L-PBF builds, possibly owing to poor flowability of powders and severe contamination. In contrast, GA and PA methods have been widely applied for spherical powder fabrication. Typical particle sizes of GA and PA powders range from 10 to $300 \mu \mathrm{m}^{24}$. These particles are further sieved to obtain a more narrow distribution. Unfortunately, these methods have limited powder yield, and are time and energy consuming. Moreover, Mo-Si-B alloys cannot be readily subjected to gas atomization due to their high melting points ${ }^{9}$, hence, there have been limited 
(a) Slurry preparation

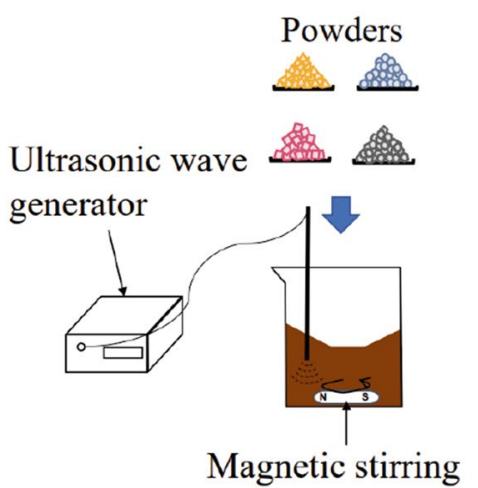

(b) Pulsated orifice ejection process

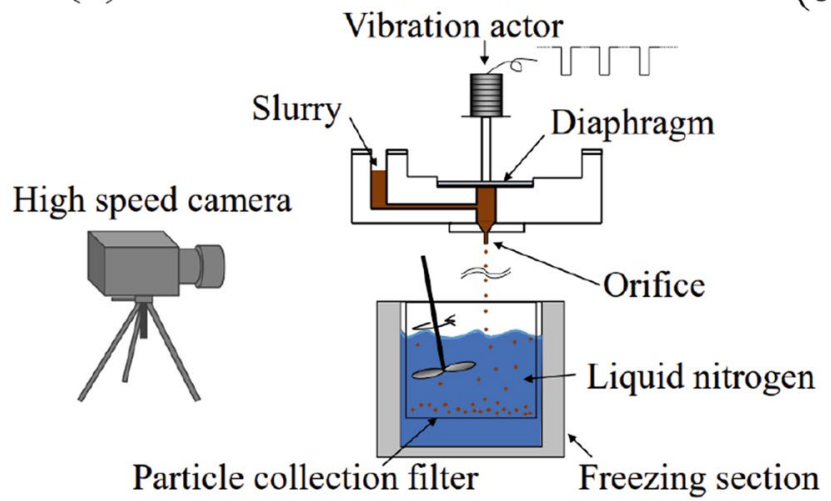

(c) Freeze-dry process

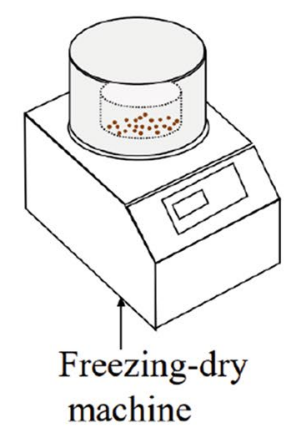

Figure 1. Schematic diagram of FD-POEM process: (a) Fabrication process of the slurry; (b) Preparation of composite particles by pulsated orifice ejection method; (c) Freeze-drying process.

studies on the fabrication of spherical powders of the Mo-Si-B alloys ${ }^{28}$. Recently, PS is a new technique that can be used for refractory powder fabrication ${ }^{29,30}$. The experimental results from Higashi et al ${ }^{30}$ showed that the PS MoSiBTiC alloy powders had a spherical shape similar to the atomized ones, while the content of elemental Ti or Si was significantly reduced owing to the ultra-high-temperature exposure. Notably, the GA, PA, PREP, and PS techniques require high-quality feedstock materials, such as wires, rods, or hydride alloy powders, further increasing the limitations. The powder manufacturing techniques for refractory materials such as MoSiBTiC alloys are still in development and not well established ${ }^{12}$.

Therefore, in order to extend the application of L-PBF to refractory alloys, it is particularly important to develop an innovative approach for powder preparation to realize a spherical shape and stable elemental composition along with high yielding and low cost. In this study, a novel technique, the freeze-dry pulsated orifice ejection method (FD-POEM), was designed to fabricate monodispersed spherical refractory particles. The working mechanism of FD-POEM, the feasibility of fabricating a high-concentration slurry, and the characteristics of the FD-POEM particles were thoroughly investigated. The present work indicates that FD-POEM process could directly fabricate spherical composite particles with arbitrary elemental ratios, dispensing with preparation of wires and rods in conventional methods. Moreover, no melting-process was performed during FD-POEM, which avoids the loss of low melting-point elements. Particularly, FD-POEM has great potential in preparing the refractory alloy or ceramic powders.

\section{Experimental}

Principle and experimental procedures of FD-POEM. The FD-POEM process involves several steps as follows: (a) slurry preparation (Fig. 1a). Mo, Si, MoB, and TiC submicron powders were selected as elemental materials and weighed according to the nominal atomic composition of 65Mo-5Si-10B-10Ti-10C (at\%) alloy. These powders were mixed in a certain amount of deionized water to form a uniform slurry with a concentration of $\mathrm{X}$ vol\% through combination of mechanical blending and ultrasonication at $273 \mathrm{~K}$ for $1 \mathrm{~h}$. Here, $\mathrm{X}$ vol\% was defined as the volume fraction of powders in the slurry. (b) Pulsed-orifice ejection process (Fig. 1b). The slurry was dropped into liquid nitrogen driven by a pulsated orifice ejection apparatus, which included a pulsated orifice ejection body, diaphragm, and orifice pipe. A diaphragm was used to separate the droplets from the orifice pipe. The outer and inner diameters of the orifice pipe are $1.0 \mathrm{~mm}$ and $0.6 \mathrm{~mm}$, respectively. The droplets were extruded from the orifice under diaphragm vibration using a pulse with a frequency of $10 \mathrm{~Hz}$, followed by a square waveform. Owing to the surface tension, the extruded droplets became spherical and then froze instantly when dropped in the liquid nitrogen. (c) Freeze drying process (Fig. 1c). Monodispersed spherical particles were obtained via complete freeze-drying for more than $24 \mathrm{~h}$.

Characterization. Zeta potential and viscosity analysis. The zeta potential of slurries was measured using a zeta potential analyzer (SZ-100, HORIBA, Ltd., JP), which utilizes a laser doppler micro-electrophoresis technique with a red laser wavelength of $633 \mathrm{~nm}$. The viscosity of slurries was evaluated using a viscosity meter (DV2T VISCOMETER, AMETEK BROOKFIELD., US). All zeta potential and viscosity measurements were conducted more than three times. To be consistent with the actual FD-POEM working environment, the zeta potential and viscosity measurements were operated at a temperature of $25^{\circ} \mathrm{C}$ in this work.

High-speed camera observation. Detailed images of the droplet ejection sequence were obtained using a highspeed camera (FASTCAM Mini UX100 type 800 K-M-8G, PHOTRON, INC., JP) with a frame rate of 4000/s and a shutter rate of $160,000 / \mathrm{s}$.

Powder feature evaluations. The particle size, distribution, and sphericity of FD-POEM particles were determined using an optical microscope (BX51, OLYMPUS Ltd., JP) equipped with an image analyzer software (Win- 

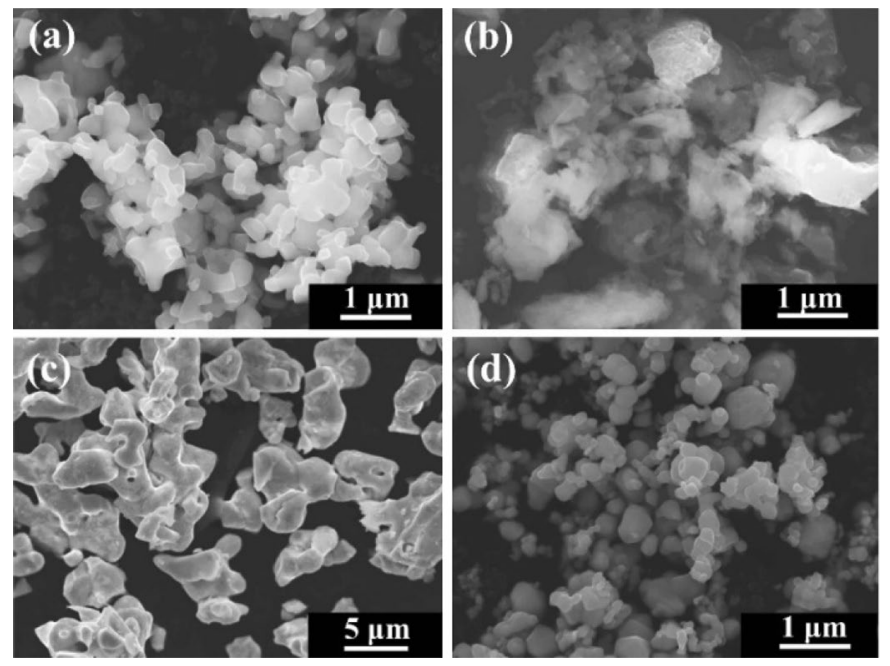

Figure 2. SEM images of the raw (a) Mo, (b) Si, (c) MoB, and (d) TiC powders.

ROOF, MITANI Ltd., JP). The sphericity of FD-POEM particles was determined according to Eq. (1) suggested by the ISO $9276-6$ standard $^{31}$.

$$
\mathrm{S}=4 \pi \mathrm{A} / \mathrm{P}^{2}
$$

where $\mathrm{S}$ is an indicator for particles' sphericity, $\mathrm{P}$ and $\mathrm{A}$ are the measured circumference and area covered by a particle projection, respectively. For completely spherical particles, the value of $S$ is equal to 1 . As the particle sphericity decreases, the $S$ value will decrease. The particle size, distribution, and sphericity were obtaining by measuring more than 200 particles.

The surface roughness of the FD-POEM particles was determined using a 3D laser scanning microscope (VK-X200 series, KEYENCE Corp., USA).

The flowability of FD-POEM particles were measured using a revolution powder analyzer (Rev2015-Revolution Powder Analyzer, Mercury Scientific Inc., USA) at $25^{\circ} \mathrm{C}$. The rotation speed was $0.3 \mathrm{rpm}$. An experimental run consisted of 150 automatically detected avalanche angles. In this work, each avalanche angle measurement was performed more than 3 times.

The microstructure and elemental distributions of FD-POEM particles were evaluated using a scanning electron microscope (JSM-6010LV, JEOL, JP). The internal structure was observed using computer tomography (CT) analyzer (TUX-3200N, MARS TOHKEN SOLUTION Co. Ltd., JP), in which a series of X-ray images was combined to construct cross-sectional morphologies of a FD-POEM powder. The samples were scanned using an $\mathrm{X}$-ray tube voltage of $74.2 \mathrm{kV}$ and tube current of $51.3 \mu \mathrm{A}$. A magnification of $45 \times$ was used to achieve $1024 \times 1024$ pixels resolution with $1.2 \mu \mathrm{m}$ pixel size. The exposure time or lamp inclined angle was $2.5 \mathrm{~s}$ or $0^{\circ}$, respectively.

\section{Result and discussion}

Fabrication of uniform slurry. The features of the raw materials are deemed to be important for FDPOEM. Figure 2 shows the morphology of the raw powders. It is noted that the particle morphologies and sizes of the raw powders differ greatly, such as polyhedral-shaped Mo and TiC powders (Fig. 2a,d), lamellar-shaped Si powder (Fig. 2b), and irregularly shaped MoB powder (Fig. 2c). According to SEM observations, the medium particle sizes, $\mathrm{d}_{50}$, of Mo, $\mathrm{Si}, \mathrm{MoB}$, and TiC powders were determined to be $\sim 1.0,4.3,0.57$, and $0.67 \mu \mathrm{m}$, respectively.

Single-component slurries of raw $\mathrm{Mo}, \mathrm{Si}, \mathrm{MoB}$, and $\mathrm{TiC}$ powders with a concentration of 5 vol\% were prepared separately. Figure $3 \mathrm{a}-\mathrm{d}$ show the appearance of different single-component slurries. Each slurry had a uniform color without obvious delamination, indicating that the raw powders were well dispersed in water. For comparison, the binary components of Mo-Si and multi-components of Mo-Si-MoB-TiC slurries were also prepared. As shown in Fig. 3e,f, the mixed slurries maintained good dispersion, and the dispersion state did not change with an increase in the amount of the slurry components.

To investigate the stability mechanism of the fabricated slurries several theoretical values that describe the particle motion in water were calculated. The ratio of $d$ (Brownian motion distance) $/ v$ (sinking velocity) is often applied to characterize the nanopowder stability in slurries. A large $d / v$ value means stable dispersion. In general, the particles can exist stably in water as the value of $d / v$ is larger than $20^{32-34}$. The sinking velocity of particles follows the Stokes' law as Eq. (2) ${ }^{35}$,

$$
v=\frac{2 r^{2}\left(\rho-\rho_{0}\right)}{9 \eta}
$$



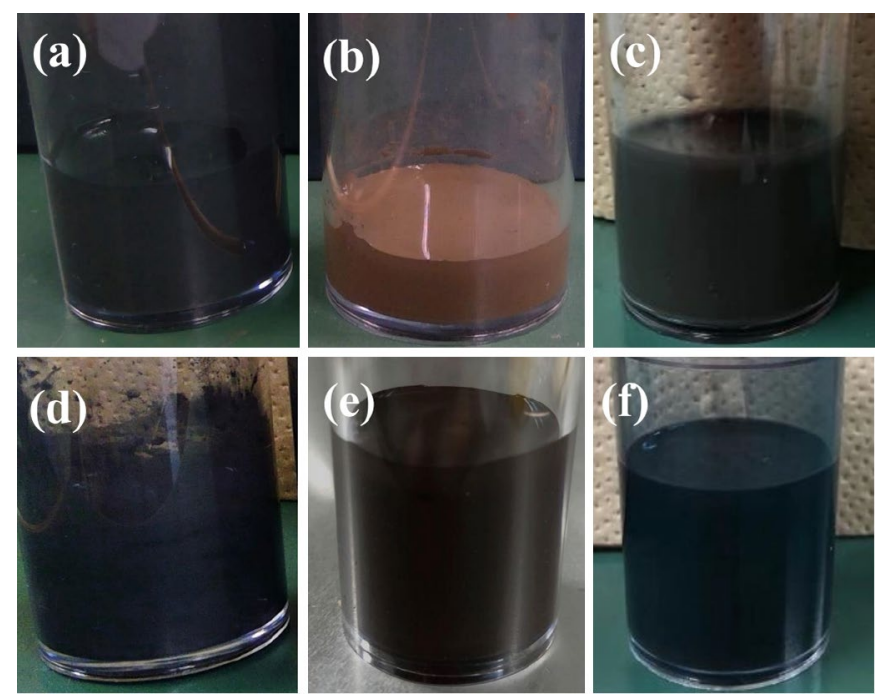

Figure 3. The appearance of $5 \mathrm{vol} \%$ (a) $\mathrm{Mo},(\mathbf{b}) \mathrm{Si}$, (c) MoB, (d) TiC, (e) Mo-Si, (f) Mo-Si-MoB-TiC slurries.

\begin{tabular}{|l|l|l|l|l|}
\hline & Mo & Si & MoB & TiC \\
\hline Viscosity, $\eta(\mathrm{mPa} s)$ & $1.03 \pm 0.05$ & $1.68 \pm 0.05$ & $0.96 \pm 0.03$ & $9.66 \pm 0.4$ \\
\hline Velocity of sinking, $\nu(\mathrm{nm} / \mathrm{s})$ & 2.15 & 0.08 & 1.78 & 0.41 \\
\hline Brownian motion, $d(\mathrm{~nm})$ & 6.75 & 9.54 & 6.75 & 8.24 \\
\hline$d / v(\mathrm{~s})$ & 3.14 & 119.25 & 3.79 & 20.10 \\
\hline
\end{tabular}

Table 1. The basic parameters of each slurry.

where $v$ is the velocity of sinking; $r$ is the diameter of particle; $\rho$ is the density of the particle; $\rho_{0}$ is density of the solution, and $\eta$ is the viscosity of the solution. The Brownian motion of powders is calculated according to the Fluctuation-dissipation theorem Eq. $(3)^{35}$,

$$
d=\sqrt{2 D t}
$$

where $t$ is the time, $d$ is the moving distance of the particle, and $D$ is the diffusion coefficient. The diffusion coefficient $D$ is expressed as Eq. (4).

$$
D=\frac{k T}{6 \pi \eta r}
$$

where $k$ is the Boltzmann constant $\left(1.38064852 \times 10^{-23} \mathrm{~J} / \mathrm{K}^{35}\right)$; $T$ is the temperature; $\eta$ is the solution viscosity, and $r$ is the particle radius. Correspondingly, the $d / v$ values are displayed in Table 1. It indicates that the slurries of $\mathrm{Si}$ and $\mathrm{TiC}$ are stable in view of the higher value of $d / v$. However, the relative lower $d / v$ values suggested that the Mo and $\mathrm{MoB}$ powders are prone to sink; obviously, this is not consistent with the experimental results shown in Fig. 3.

We believe that the discrepancy between the theoretical values and the experimental data shown in Table 1 could be explained by the presence of a repulsive force between particles in the slurry. Herein, the surface electrostatic repulsive force between neighbouring particles is considered, which can be reflected by the zeta potential measurements. It is documented in the literature that when the absolute value of the zeta potential exceeds $30 \mathrm{mV}$, the particle suspension is in a stable state. As shown in Fig. 4, the absolute zeta potential values of all powders in our experiments were greater than $30 \mathrm{mV}$, indicating that all powders exerted strong repulsive forces in the slurry. Based on the aforementioned, we concluded that the natural motion and surface charge of powders synergistically contributed to the remarkable stability of the slurries. Because each type of raw powder had the same negative charge, the heterogeneous deposition phenomenon did not occur during the fabrication of the hybrid slurries.

The slurry viscosity is a critical parameter required for the FD-POEM process. Thus, the viscosities of various powder slurries were investigated at room temperature. As shown in Fig. 5, the slurry viscosity exhibits a linear increasing trend with an increase in the slurry concentration. In addition, the range of slurry viscosity that is suitable for FD-POEM was determined. The dotted line in Fig. 5 indicates the viscosity limit of $50 \mathrm{mPa}$ for the operation. When the viscosity exceeds $50 \mathrm{mPa}$ s, the slurry cannot be ejected from the orifice, regardless of the diaphragm movement. 


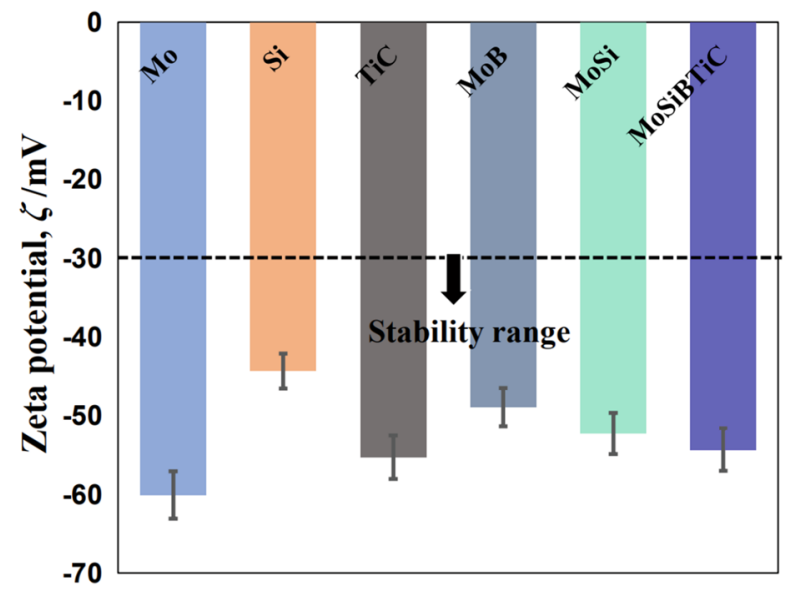

Figure 4. The zeta potential of various powders dispersed in water.

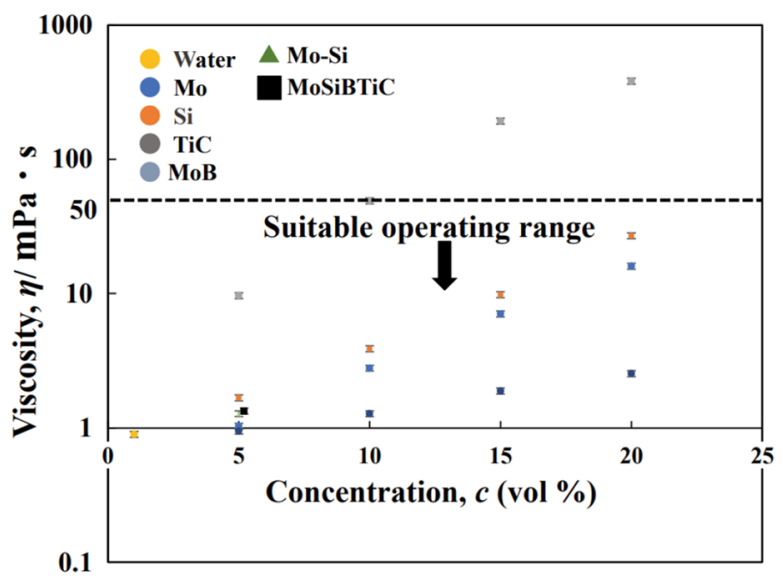

Figure 5. The slurry concentration dependent of the slurry viscosity.

Fabrication and evaluation of FD-POEM particles. Single-component FD-POEM particles. Singlecomponent powders of $\mathrm{Mo}, \mathrm{Si}, \mathrm{MoB}$, and $\mathrm{TiC}$ were fabricated individually. The typical morphologies are shown in Fig. 6. As shown in Table 2 and Fig. 6, all FD-POEM particles were nearly spherical with a sphericity value over 0.8 , displaying a rough surface. This spherical shaped morphology is beneficial for the powder flow. Many internal pores were present in the FD-POEM particles. The formation mechanism of pores is closely related to the nature of FD-POEM, which will be discussed in "Formation mechanism of pores in FD-POEM particles" section. The surface features of the various FD-POEM particles exhibit significant differences. As shown in Table 2, the Mo and MoB FD-POEM particles exhibited higher surfaces roughness $(\mathrm{Ra})$ than that of Si and TiC particles, agreeing with the SEM observations in Fig. 6 . This difference was possibly caused by the size difference of the raw powders. These FD-POEM particles are composed of raw nano or micro-sized particles. First, the small nanoparticles are prone to rearrange and stack owing to the surface tension created during the pulsated orifice ejection process. Second, the interaction between small particles is greater than that of large particles, according to the negative correlation between the van der Waals force and the size of the particles ${ }^{36,37}$. The $\mathrm{Si}$ and TiC FD-POEM particles consisting of smaller raw powders exhibited stronger cohesion forces compared to those of Mo and MoB, thereby decreasing the surface roughness.

Binary-component FD-POEM particles. To verify the feasibility of preparing arbitrary-component particles by FD-POEM, Mo-Si composite particles were fabricated with various elemental ratios. Mo- 10 at $\% \mathrm{Si}$, Mo- 30 at $\% \mathrm{Si}$, Mo-50at $\% \mathrm{Si}$ and Mo-70at\%Si composite slurries with a concentration of 5 vol\% were prepared and processed by FD-POEM, respectively. As shown in Fig. 7, as the Si content increased, the surface of the FD-POEM particles became denser and smoother. Correspondingly, the Ra values decreased from 10.8 to $2.3 \mu \mathrm{m}$ (Table 2). However, the sizes of the Mo-Si FD-POEM particles, $d_{50}$, are similar, in the range of $730-780 \mu \mathrm{m}$. This indicates that the composition and concentration of the slurry have an insignificant influence on the particle size of the FD-POEM particles. Therefore, spherical FD-POEM particles with arbitrary composition ratios can be prepared by FD-POEM. 

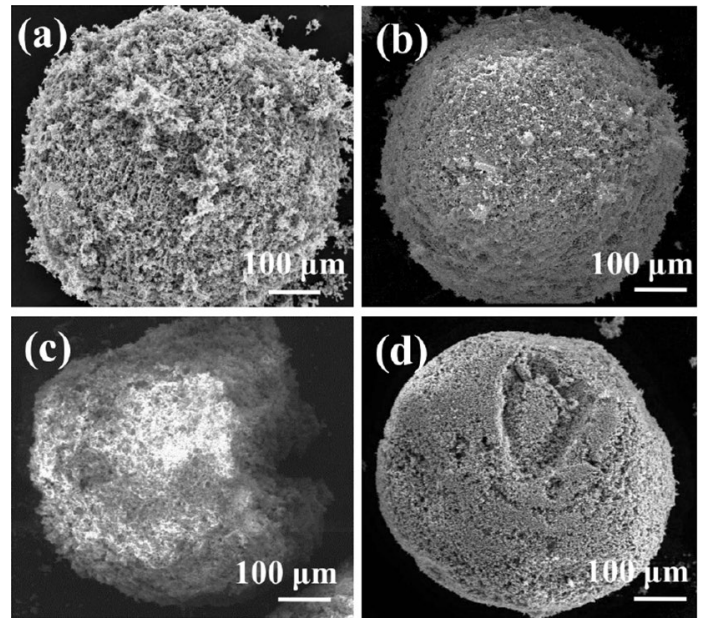

Figure 6. SEM images of the monodispersed single-component (a) Mo, (b) Si, (c) MoB, (d) TiC particles by FD-POEM.

\begin{tabular}{|c|c|c|c|c|c|c|c|c|c|}
\hline & Mo & $\mathbf{S i}$ & MoB & $\mathrm{TiC}$ & Mo- $10 \% S i$ & Mo-30\%Si & Mo-50\%Si & Mo-70\%Si & $\begin{array}{l}\text { Mo-Si-MoB- } \\
\text { TiC }\end{array}$ \\
\hline $\begin{array}{l}\text { Surface } \\
\text { roughness } \\
(R a, \mu \mathrm{m})\end{array}$ & $28.3 \pm 5.1$ & $6.4 \pm 1.5$ & $36.1 \pm 6.7$ & $4.4 \pm 1.7$ & $10.8 \pm 2.2$ & $7.7 \pm 1.2$ & $3.5 \pm 2.0$ & $2.3 \pm 0.7$ & $5.6 \pm 1.8$ \\
\hline Sphericity & $0.86 \pm 0.02$ & $0.93 \pm 0.01$ & $0.81 \pm 0.02$ & $0.88 \pm 0.01$ & $0.90 \pm 0.01$ & $0.95 \pm 0.01$ & $0.94 \pm 0.01$ & $0.94 \pm 0.01$ & $0.94 \pm 0.01$ \\
\hline
\end{tabular}

Table 2. The surface roughness $(\mathrm{Ra})$ and sphericity values of different FD-POEM particles.
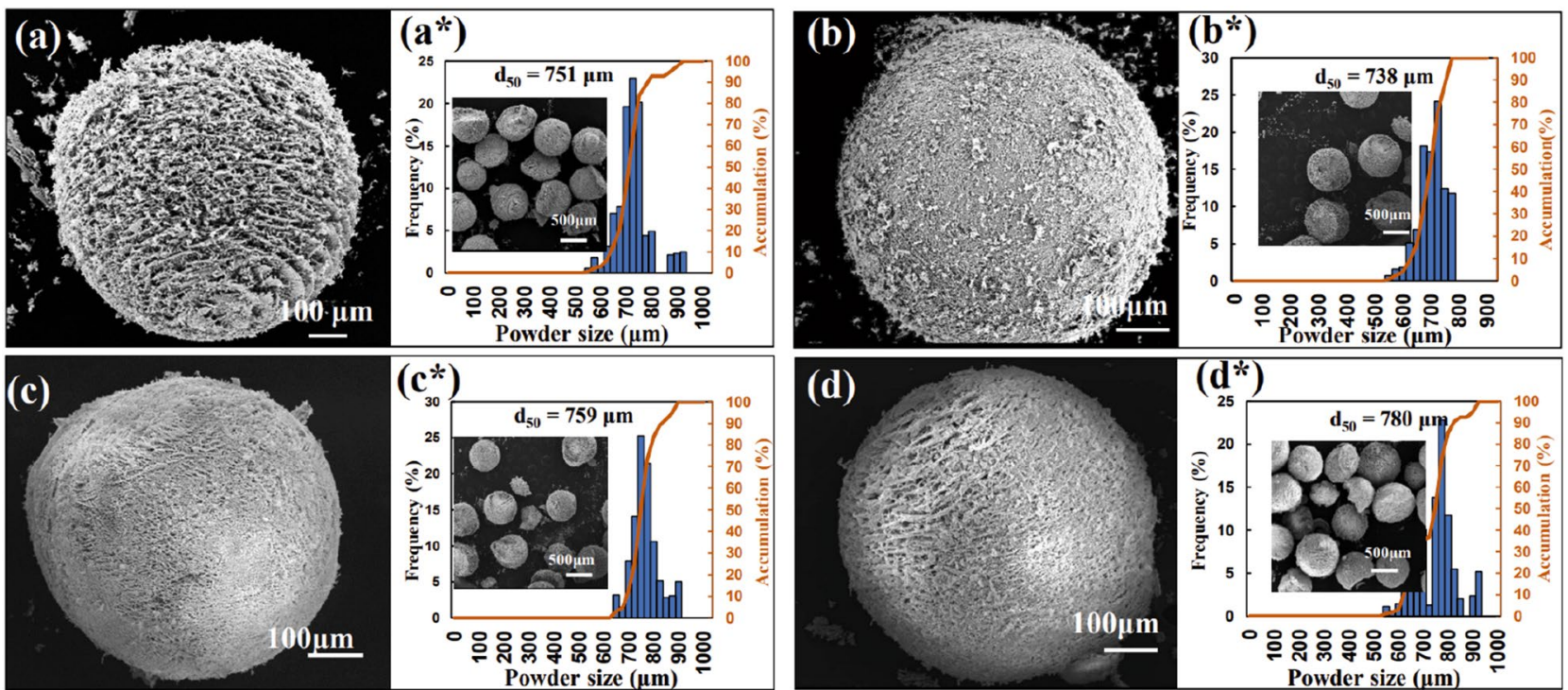

Figure 7. The SEM images and size distribution of the FD-POEM particles (a: Mo-10at\%Si; b: Mo-30at\%Si; c: Mo-50at\%Si; d: Mo-70at\%Si). The inserts were low-magnification microphotographs.

Multicomponent FD-POEM particles. A spherical multi-component particle of MoSiMoTiC was also prepared (see Fig. 8a). As confirmed by SEM-EDS mappings (see Fig. 8b-e), elemental Mo, Si, and Ti were evenly distributed on the particle surface. Figure $8 \mathrm{f}$ shows the particle size distribution of the MoSiBTiC particles fabricated by FD-POEM. It can be seen that the size distribution is within a narrow range and follows a typical Gaussian curve. The particle size $\mathrm{d}_{50}$ was determined to be $745 \mu \mathrm{m}$.

In addition, the powder flowability of FD-POEM particles was reflected by a revolution method, where lower avalanche angles indicated better flowability. The avalanche angle of the FD-POEM MoSiBTiC particles 

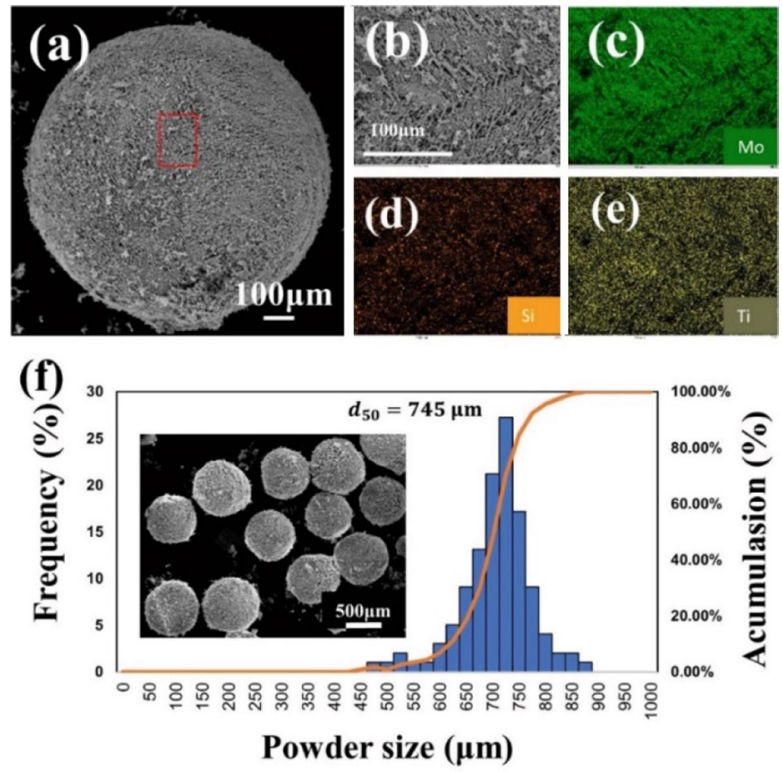

Figure 8. (a) SEM images of Mo-Si-MoB-TiC particles by FD-POEM; (b-e) the enlarged area marked in red box in (a) and corresponding EDS mappings; (f) the size distribution and low-magnification SEM image of Mo-Si-MoB-TiC particles.
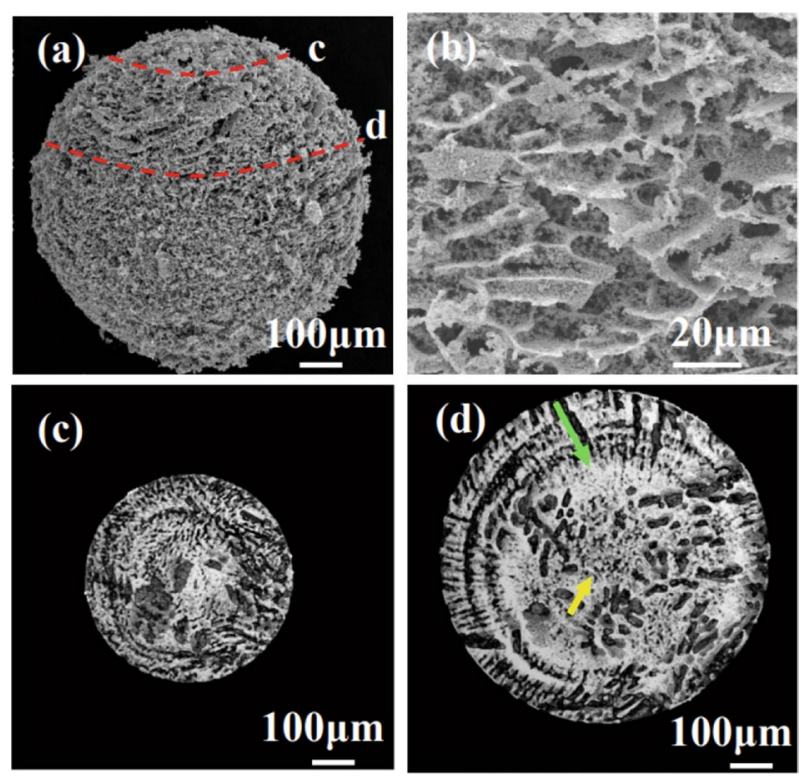

Figure 9. (a) low-magnification and (b) high-magnification SEM images of a Mo-30at\%Si FD-POEM particle. (c,d) The X-ray CT images of the areas marked by red lines in (a).

was measured to be $42.6 \pm 3^{\circ}$, which is lower than that of the ball-milled alloy powders $\left(\sim 50.5^{\circ}\right)$. This flowability improvement was attributed to an excellent sphericity $(\sim 0.94)$ of FD-POEM particles.

Notably, this is the first time that refractory composite particles were fabricated directly without a melting process.

Formation mechanism of pores in FD-POEM particles. To investigate the internal structure of the FD-POEM particles, we performed high-magnification observations using a field emission scanning electron microscope (FE-SEM). Figure 9a,b show low and high magnifications, respectively of an FD-POEM Mo-30at\%Si particle. This particle showed porous structures with flaky topography. The porous feature was further confirmed by cross-sectional X-ray CT as shown in Fig. 9c,d taken from the red line in Fig. 9a. It is noted that the micro-sized voids with radial dendrites (green arrow in Fig. 9d) extend from the outside to the inside. Moreover, some smaller voids were observed in the central area of the particle (yellow arrow in Fig. 9d). These voids were 


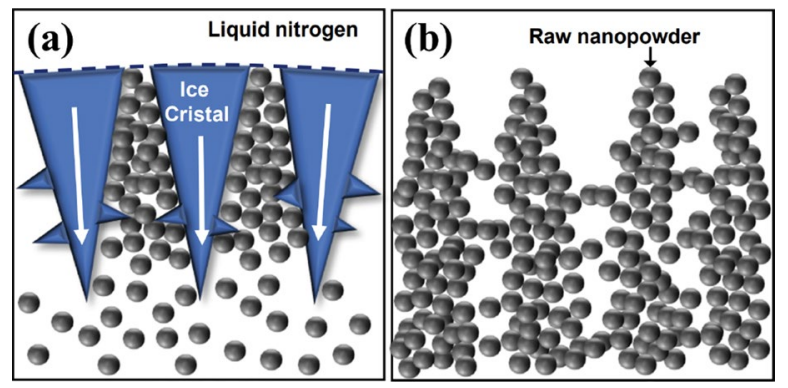

Figure 10. (a) Schematic diagrams of (a) ice crystal growth during the freezing process and (b) ice sublimation during the freeze-drying process. The white arrows in (a) indicate the direction of ice crystal growth.

formed during the freezing and drying processes. Once the slurry encounters liquid nitrogen, ice crystallization starts driven by a temperature gradient. The raw powders were rejected by the ice crystal growth and concentrated between the ice crystals, as illustrated in Fig. 10a.

The distribution and movement of nanopowders in the ice crystals were influenced by thermodynamics during crystallization resulting in the formation of voids. Subsequently, the ice crystals containing nanoparticles were exposed to the continuous cooling. The pores were generated further from the sublimation of the ice crystals, leading to the radial distribution of voids from the outside to the inside of the particle (Fig. 10b). Therefore, the spherical FD-POEM particles exhibited a mesh-pored structure following the track of the sublimated ice crystals.

In general, the porosity $p$ is a parameter used to evaluate the quality of particles. Here, the porosity value of the FD-POEM particles can be calculated based on Eq. (5):

$$
p=1-\frac{\frac{\omega_{1} \times m}{\rho_{1}}+\frac{\omega_{2} \times m}{\rho_{2}}+\cdots+\frac{\omega_{i} \times m}{\rho_{i}}}{V}
$$

where $\omega_{i}, \rho$ are the mass fraction and density of component $i$, respectively. $m$ and $V$ are the weight and volume of an FD-POEM particle, respectively. Correspondingly, the actual porosity was calculated to be $93.2 \pm 5$ vol $\%$. This value is consistent with the water volume fraction in the $95 \mathrm{vol} \%$. In contrast, the porosity was measured to be $\sim 14.0$ vol\% from the X-ray CT analysis in Fig. 9c,d, which is much lower than the actual value, attributed to the limited resolution of X-ray CT $(\sim 3.1 \mu \mathrm{m})$. This result indicates that $\sim 80 \%$ of the pores in the PD-POEM particles have a small size $(<3.1 \mu \mathrm{m})$. To prove the applicability of FD-POEM particles for L-PBF, a MoSiBTiC build (see Fig. S1 of Supplementary Information) was fabricated successfully using an in-house-developed L-PBF equipment.

In the near future, our main work will focus on the control of FD-POEM particle size by adjusting the pipe orifice size and vibration movement of the diaphragm. The microstructure and mechanical performance of L-PBF parts using FD-POEM particles will also be investigated.

\section{Conclusion}

In this study, a novel powder preparation method, FD-POEM, was systematically developed to fabricate refractory alloy composite particles. The main conclusions are summarized as follows:

(1) High-concentration, stable slurries were prepared by dispersing submicron $\mathrm{Mo}, \mathrm{Si}$, MoB, and TiC powders in water. It was found that the viscosity of the slurry should be lower than $50 \mathrm{mPa}$ s to meet the operating requirements of FD-POEM.

(2) Spherical composite particles with arbitrary composition ratios could be fabricated by FD-POEM. The particles had a narrow size range and uniform elemental distribution. Moreover, many mesh pores $\sim 93.2 \%$ in volume were formed within the FD-POEM fabricated particles, which was attributed to the ice crystal freezing process and subsequent ice sublimation.

(3) Owing to their spherical morphology, the FD-POEM particles had a low avalanche angle of $42.6^{\circ}$, exhibiting good flowability. This study offers new insights into the production of complex system refractory particles, such as MoSiBTiC alloy particles and high-entropy alloy particles for L-PBF.

Received: 20 May 2021; Accepted: 23 July 2021

Published online: 16 August 2021

\section{References}

1. Perepezko, J. H. The hotter the engine, the better. Science 326, 1068-1069 (2009).

2. Senkov, O. et al. Microstructure and elevated temperature properties of a refractory TaNbHfZrTi alloy. J. Mater. Sci. 47, 4062-4074 (2012). 
3. Jéhanno, P., Heilmaier, M. \& Kestler, H. Characterization of an industrially processed Mo-based silicide alloy. Intermetallics 12, 1005-1009 (2004).

4. Yoshimi, K. et al. High-temperature compressive properties of TiC-added Mo-Si-B alloys. JOM 66, 1930-1938 (2014).

5. Nakamura, J., Kanekon, D. \& Yoshimi, K. Characterization of Mo/Mo2C interface in MoSiBTiC alloy. Mater. Lett. 180, 340-343 (2016).

6. Kamata, S. Y. et al. Ultrahigh-temperature tensile creep of TiC-reinforced Mo-Si-B-based alloy. Sci. Rep. 8, 1-14 (2018).

7. Moriyama, T. et al. Room-temperature fracture toughness of MoSiBTiC alloys. Intermetallics 84, 92-102 (2017).

8. Uemura, S. et al. Quantitative evaluation of microstructure in Mo-Si-B-TiC alloy produced by melting and tilt casting methods. Mater. Trans. 59, 136-145 (2018).

9. Fukuyama, H., Sawada, R., Nakashima, H., Ohtsuka, M. \& Yoshimi, K. Study of solidification pathway of a MoSiBTiC alloy by optical thermal analysis and in-situ observation with electromagnetic levitation. Sci. Rep. 9, 1-11 (2019).

10. Lemberg, J. \& Ritchie, R. Mo-Si-B alloys for ultrahigh-temperature structural applications. Adv. Mater. 24, 3445-3480 (2012).

11. Dimiduk, D. M. \& Perepezko, J. H. Mo-Si-B alloys: Developing a revolutionary turbine-engine material. MRS Bull. 28, 639-645. https://doi.org/10.1557/mrs2003.191 (2003).

12. Zhou, W. et al. Powder fabrication and laser additive manufacturing of MoSiBTiC alloy. Intermetallics 104, 33-42 (2019).

13. Abe, F., Osakada, K., Shiomi, M., Uematsu, K. \& Matsumoto, M. The manufacturing of hard tools from metallic powders by selective laser melting. J. Mater. Process. Technol. 111, 210-213 (2001).

14. Kruth, J.-P. et al. Selective laser melting of iron-based powder. J. Mater. Process. Technol. 149, 616-622 (2004).

15. Zhao, X., Chen, J., Lin, X. \& Huang, W. Study on microstructure and mechanical properties of laser rapid forming Inconel 718. Mater. Sci. Eng., A 478, 119-124 (2008).

16. Thijs, L., Verhaeghe, F., Craeghs, T., Humbeeck, J. V. \& Kruth, J.-P. A study of the microstructural evolution during selective laser melting of Ti-6Al-4V. Acta Mater. 58, 3303-3312 (2010).

17. Ali, U. et al. On the measurement of relative powder-bed compaction density in powder-bed additive manufacturing processes. Mater. Des. 155, 495-501 (2018).

18. Olakanmi, E. O., Cochrane, R. F. \& Dalgarno, K. W. A review on selective laser sintering/melting (SLS/SLM) of aluminium alloy powders: Processing, microstructure, and properties. Prog. Mater Sci. 74, 401-477. https://doi.org/10.1016/j.pmatsci.2015.03.002 (2015).

19. Liu, C. et al. Fabrication of micro-fine spherical Ti-6Al-4V alloy powders based on hydrogen decrepitation and plasma spheroidisation. Powder Metall. 59, 229-235 (2016).

20. Song, B., Dong, S., Deng, S., Liao, H. \& Coddet, C. Microstructure and tensile properties of iron parts fabricated by selective laser melting. Opt. Laser Technol. 56, 451-460 (2014).

21. Zhou, W. et al. Interfacial reaction induced efficient load transfer in few-layer graphene reinforced Al matrix composites for highperformance conductor. Compos. B Eng. 167, 93-99 (2019).

22. Li, Y. \& Gu, D. Parametric analysis of thermal behavior during selective laser melting additive manufacturing of aluminum alloy powder. Mater. Des. 63, 856-867 (2014).

23. Takaichi, A. et al. Microstructures and mechanical properties of $\mathrm{Co}-29 \mathrm{Cr}-6 \mathrm{Mo}$ alloy fabricated by selective laser melting process for dental applications. J. Mech. Behav. Biomed. Mater. 21, 67-76 (2013).

24. Sun, P., Fang, Z. Z., Zhang, Y. \& Xia, Y. Review of the methods for production of spherical Ti and Ti alloy powder. Jom 69, 1853-1860 (2017).

25. McCracken, C. G., Motchenbacher, C. \& Barbis, D. P. Review of titanium-powder-production methods. Int. J. Powder Metall. 46, $16-26$ (2010).

26. Spierings, A. B., Voegtlin, M., Bauer, T. U. \& Wegener, K. Powder flowability characterisation methodology for powder-bed-based metal additive manufacturing. Prog. Addit. Manuf. 1, 9-20 (2016).

27. Zhou, W., Tsunoda, K., Nomura, N. \& Yoshimi, K. Effect of hot isostatic pressing on the microstructure and fracture toughness of laser additive-manufactured MoSiBTiC multiphase alloy. Mater. Design 196, 109132 (2020).

28. Schmelzer, J. et al. Printability of gas atomized Mo-Si-B powders by laser metal deposition. Int. J. Refract Metal Hard Mater. 78, $123-126$ (2019).

29. Boulos, M. Plasma power can make better powders. Met. Powder Rep. 59, 16-21 (2004).

30. Higashi, M. \& Ozaki, T. Selective laser melting of MoSiBTiC alloy with plasma-spheroidized powder: Microstructure and mechanical property. Mater. Charact. 172, 110888 (2021).

31. ISO, I. 9276-6 Representation of Results of Particle Size Analysis-Part 6: Descriptive and Quantitative Representation of Particle Shape and Morphology (ISO, 2008).

32. Upadhyay, R. K. \& Kumar, A. Boundary lubrication properties and contact mechanism of carbon/MoS2 based nanolubricants under steel/steel contact. Colloid Interface Sci. Commun. 31, 100186 (2019).

33. Simha, R. The influence of Brownian movement on the viscosity of solutions. J. Phys. Chem. 44, 25-34 (1940).

34. Felderhof, B. \& Jones, R. Linear response theory of the viscosity of suspensions of spherical brownian particles. Phys. A 146, 417-432 (1987).

35. Dhont, J. K. An Introduction to Dynamics of Colloids (Elsevier, 1996).

36. Parteli, E. J. et al. Attractive particle interaction forces and packing density of fine glass powders. Sci. Rep. 4, 1-7 (2014).

37. Chen, H., Wei, Q., Wen, S., Li, Z. \& Shi, Y. Flow behavior of powder particles in layering process of selective laser melting: Numerical modeling and experimental verification based on discrete element method. Int. J. Mach. Tools Manuf 123, 146-159 (2017).

\section{Acknowledgements}

This research was partially supported by the JST-MIRAI Program, Grant Number JPMJMI17E7 in Japan.

\section{Author contributions}

Z.Z. contributed on designing the experiment, performing the fabrication and analyzing the results. S.G. and W.Z. analyzed the results. N.N. supervised the research.

\section{Competing interests}

The authors declare no competing interests.

\section{Additional information}

Supplementary Information The online version contains supplementary material available at https://doi.org/ 10.1038/s41598-021-96187-w.

Correspondence and requests for materials should be addressed to W.Z. or N.N.

Reprints and permissions information is available at www.nature.com/reprints. 
Publisher's note Springer Nature remains neutral with regard to jurisdictional claims in published maps and institutional affiliations.

(c) (i) Open Access This article is licensed under a Creative Commons Attribution 4.0 International License, which permits use, sharing, adaptation, distribution and reproduction in any medium or format, as long as you give appropriate credit to the original author(s) and the source, provide a link to the Creative Commons licence, and indicate if changes were made. The images or other third party material in this article are included in the article's Creative Commons licence, unless indicated otherwise in a credit line to the material. If material is not included in the article's Creative Commons licence and your intended use is not permitted by statutory regulation or exceeds the permitted use, you will need to obtain permission directly from the copyright holder. To view a copy of this licence, visit http://creativecommons.org/licenses/by/4.0/.

(C) The Author(s) 2021 